\title{
Use of tissue expanders in adult postpneumonectomy syndrome
}

\author{
Anne Floor M. Macaré van Maurik, MD, a,d Bart M. Stubenitsky, MD, PhD, ${ }^{d}$ Henry A. van Swieten, MD, PhD, MsSc, \\ Vincent A. M. Duurkens, MD, ${ }^{c}$ Erik Laban, MD, and Moshe Kon, MD, PhD ${ }^{d}$
}

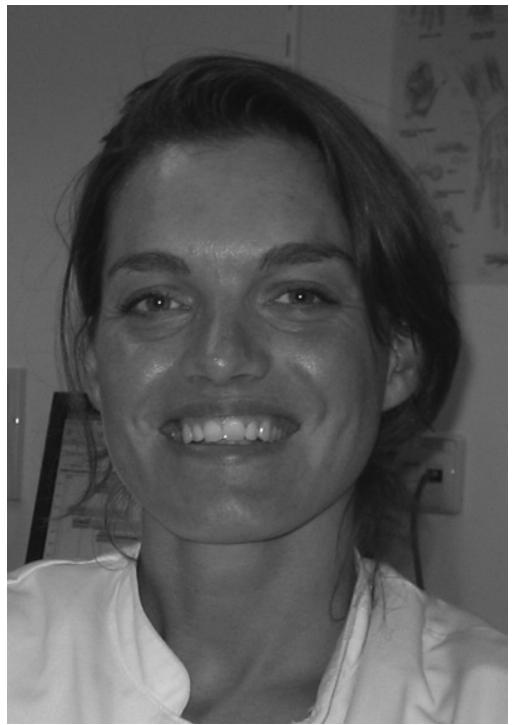

Dr Macaré van Maurik
Objective: Mediastinal shift and rotation after pneumonectomy can lead to severe symptomatic airway compression. Historically, a variety of treatments, such as muscle-flap transposition, pericardial fixation, and plombage, have been used. In this study we retrospectively evaluated the effectiveness of intrathoracic tissue expansion in postpneumonectomy syndrome.

Methods: Since 1990, our center has used tissue expanders as plombage in patients with postpneumonectomy syndrome. Between 1990 and 2005, a total of 20 patients were treated. The outcome was evaluated by using preoperative, perioperative, and postoperative bronchoscopy and imaging studies. Patient satisfaction was determined with a validated questionnaire.

Results: In 19 of the 20 patients, up to 3 tissue expanders were placed and filled within the pleural cavity. Access to the pleural cavity could not be obtained in 1 patient because of adhesions. Perioperative and postoperative bronchoscopic scans demonstrated decompression of the left main bronchus in $16(84 \%)$ of 19 patients. On discharge, all patients reported improvement of their respiratory symptoms. Six (32\%) patients required reoperation because of herniation $(n=2)$, luxation $(n=1)$, inadequate positioning $(\mathrm{n}=2)$, and leakage of the tissue expander $(\mathrm{n}=4)$. In 4 patients additional filling was performed in the outpatient clinic, with immediate improvement of respiratory distress.

Conclusion: Use of tissue expanders in adults with postpneumonectomy syndrome is an effective means of decompressing the remaining bronchus, thereby leading to a significant improvement in respiratory symptoms. Although $32 \%$ of patients required reoperation for complications, each complication was readily correctable.

$\mathrm{T}$ he postpneumonectomy syndrome (PPS), a rare late complication of pneumonectomy, is characterized by severe shift and rotation of the mediastinum to the pneumonectomy side, which leads to stretching and compression of the remaining bronchus. Patients have progressive dyspnea, stridor, and recurrent pulmonary infections. Without intervention, the syndrome leads to respiratory failure and death.

The syndrome has mostly been documented in children, presumably because of increased mobility of the mediastinum, elasticity of the lung, and compressibility of the bronchi. ${ }^{1}$ In adults PPS occurs to a much lesser degree.

In the past, many treatments have been used for PPS, including pericardial fixation, intrathoracic plombage, and muscle-flap transposition. ${ }^{2}$ Today, the surgical procedure of choice consists of placement of expandable Silastic prostheses (Dow Corning) within the empty hemithorax to redirect the mediastinum to the middle and alleviate the bronchial compression.

We present our experience involving 20 adults treated for PPS at our center between 1990 and 2005. In all cases treatment consisted of extrapleural implantation of expandable prostheses. 


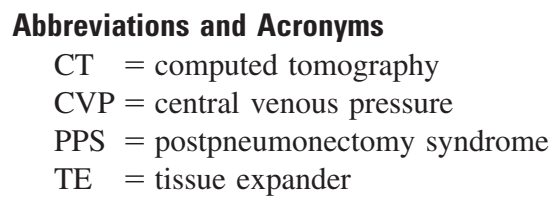

Abbreviations and Acronyms

$\mathrm{CT}=$ computed tomography

$\mathrm{CVP}=$ central venous pressure

PPS = postpneumonectomy syndrome

$\mathrm{TE}=$ tissue expander

\section{Materials and Methods}

We performed a retrospective review of adult patients with PPS who were treated with implanted tissue expanders (TEs) at our institution between 1990 and 2005. PPS was diagnosed based on the results of chest radiography, computed tomography (CT), and bronchoscopy. The institutional review board waived the need for consent.

Eurosilicone Tissue Expanders (ES12, $750 \mathrm{~mL}$ ) were used.

\section{Insertion of the Expandable Prosthesis}

Patients were operated on after achievement of general anesthesia. A short segment of the original thoracotomy, just large enough to insert the deflated TEs, was reopened. In several cases this initiated spontaneous movement of the mediastinum in a more central position, after which the volume of saline to fill the pleural space was measured. In the other cases, an extrapleural space to insert the TEs (1-3) was created, and inflation was slowly started. The mediastinum was not surgically centralized; the pressure of the prosthesis induced the movement of the mediastinum. Blood loss was measured during the procedure.

\section{Perioperative and Postoperative Imaging Studies}

During the procedure, the central venous pressure (CVP) was measured directly by using a CVP monitor. Bronchoscopy was performed during and 1 week after surgical intervention to evaluate the degree of decompression of the left bronchus. Chest

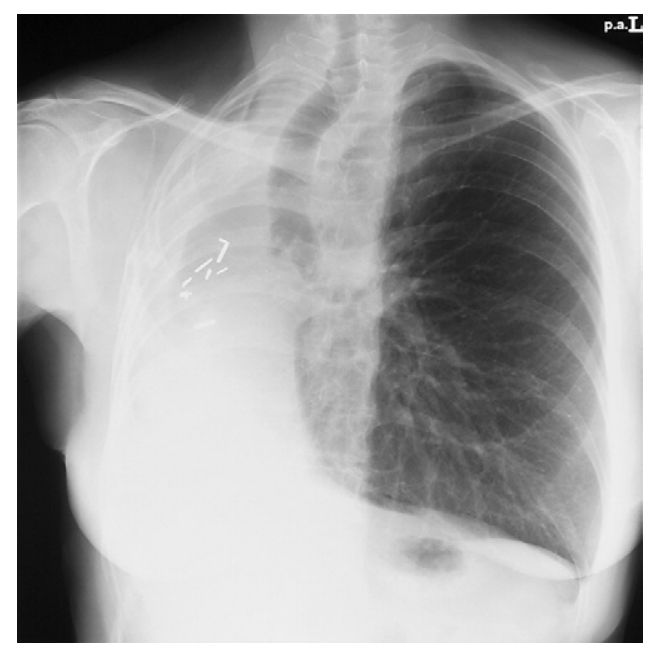

Figure 1. Chest $x$-ray film before implantation of the expandable prosthesis demonstrates a shift of the trachea and the mediastinum in a patient.

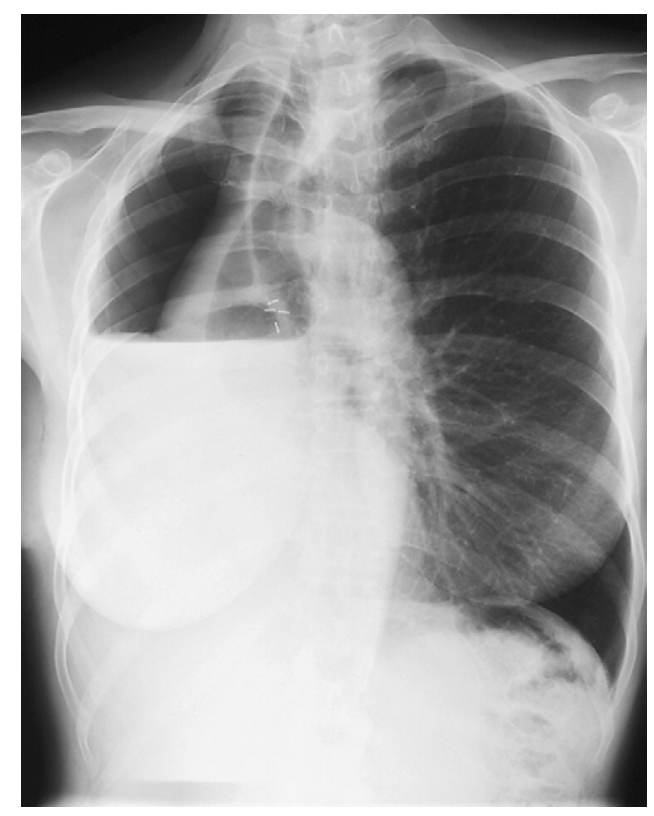

Figure 2. Chest $\mathrm{X}$-ray film before implantation of the expandable prosthesis demonstrates a shift of the trachea and the mediastinum in another patient.

radiographs and CT scans were repeated 1 week postoperatively for comparison with preoperative imaging.

\section{Postoperative Evaluation}

Because most patients were referred from other centers, follow-up was mostly carried out by means of patient questionnaire, evaluating level of functioning, degree of stridor, and patient condition. Questionnaire validation was done by using a pilot study, in which the same method was used in the same type of patients. Responses were checked for construct validity, including confirmatory factor analysis to check the factor structure of the scale, and international reliability. Cronbach values were between 0.74 and 0.95 , thus demonstrating validity.

\section{Results}

Patients

The manifestation of PPS was between 4 months and 15 years after pneumonectomy for primary lung cancer or metastasis. The syndrome followed right pneumonectomy in 19 patients and left pneumonectomy with a right aortic arch in 1 patient. Seven of the patients were men. Age at the time of PPS treatment ranged between 35 and 63 years.

\section{Preoperative Imaging Studies}

Chest radiographs of the patients after right pneumonectomy showed a deviated trachea and a crossing of the left bronchus from the left to the right in relation to the spine. Hyperinflation of the left lung with consequent shift of the heart and the great vessels was observed (Figures 1 and 2). 
Postpneumonectomy CT imaging confirmed the above findings. Bronchoscopic imaging revealed displacement of the trachea and frequently a lateral wall impression of the aortic arch and the trachea, causing compression.

\section{Insertion of the Expandable Prosthesis}

The volume of saline required to fill the expanders was 200 to $1750 \mathrm{~mL}$. Filling was stopped when the CVP increased by more than $5 \mathrm{~mm} \mathrm{Hg}$ or was greater than $10 \mathrm{~mm} \mathrm{Hg}$. In all but 1 patient this resulted in a repositioning of the mediastinum without important hemodynamic disturbances. The filling port was placed subcutaneously in a posterolateral position. In 1 patient access to the pleural cavity could not be obtained because of severe adhesions. There was no significant blood loss $(<750 \mathrm{~mL})$.

\section{Perioperative and Postoperative Imaging Studies}

Insertion of the TEs led to rapid clinical improvement in 17 (90\%) of the 19 patients. In these patients postoperative radiographs revealed midline or near-midline repositioning. Peroperative bronchoscopy visualized immediate decompression of the left main bronchus in 16 (84\%) of the 19 patients. Additional filling postoperatively resulted in decompression of the main bronchus in 1 of the 3 patients who did not have adequate decompression of the main bronchus initially.

In 6 (32\%) of 19 patients, 9 complications were observed between 2 weeks and 5 years after surgical intervention: herniation ( $\mathrm{n}=2)$, luxation $(\mathrm{n}=1)$, inadequate positioning $(n=2)$, and leakage of the TE $(n=4$; Table 1$)$. In 1 of the patients with a herniated TE, replacement and primary closure resulted in a recurrence.

Inadequate positioning of the TEs resulted in suboptimal decompression of the left main bronchus in 2 patients (Figure 3). Eventually, the clinical condition of both patients was clearly improved after repositioning and replacement of the expander.

In 1 of the 4 patients requiring surgical intervention for leakage of a TE, a recurrence occurred, leading to a renewed intervention. In only 1 case was the TE luxated behind the heart 1 month after placement, leading to symptoms of dysphagia. Additional examination by means of CT confirmed the shift of the prosthesis and mediastinum, which was solved by means of surgical release of the mediastinum through sternotomy and replacement of the TE (Figure 4).

TABLE 1. Complications after plombage of the pleural cavity with tissue expanders

\begin{tabular}{lccc}
\hline Complication & Prevalence & No. of patients & Period after operation \\
\hline Luxation & 1 & 1 & $2 \mathrm{wk}$ \\
Malposition & 2 & 1 & $3 \mathrm{wk}$ \\
Herniation & 2 & 1 & $7-9 \mathrm{mo}$ \\
Leakage & 4 & 3 & $3 \mathrm{mo}-5 \mathrm{y}$ \\
\hline
\end{tabular}

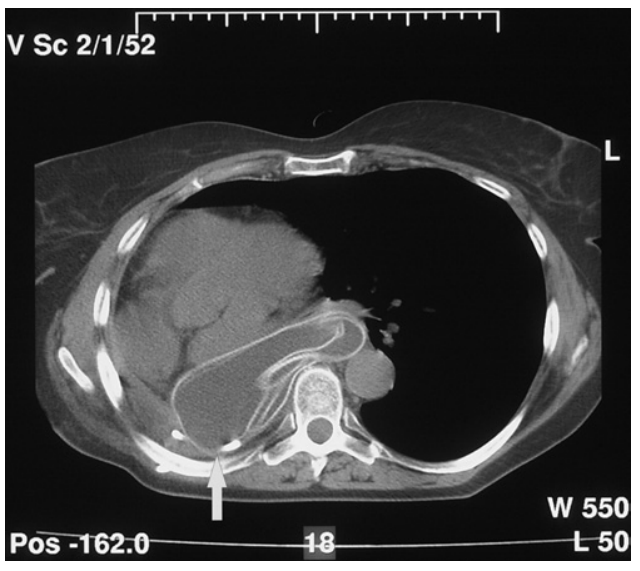

Figure 3. Postoperative computed tomographic scan of the chest demonstrates the inadequate position of the tissue expander (arrow).

Spontaneous movement of the mediastinum to a more central position after reopening the original thoracotomy was more often seen in patients with PPS up to 1 year after pneumonectomy. In the same group, complications,
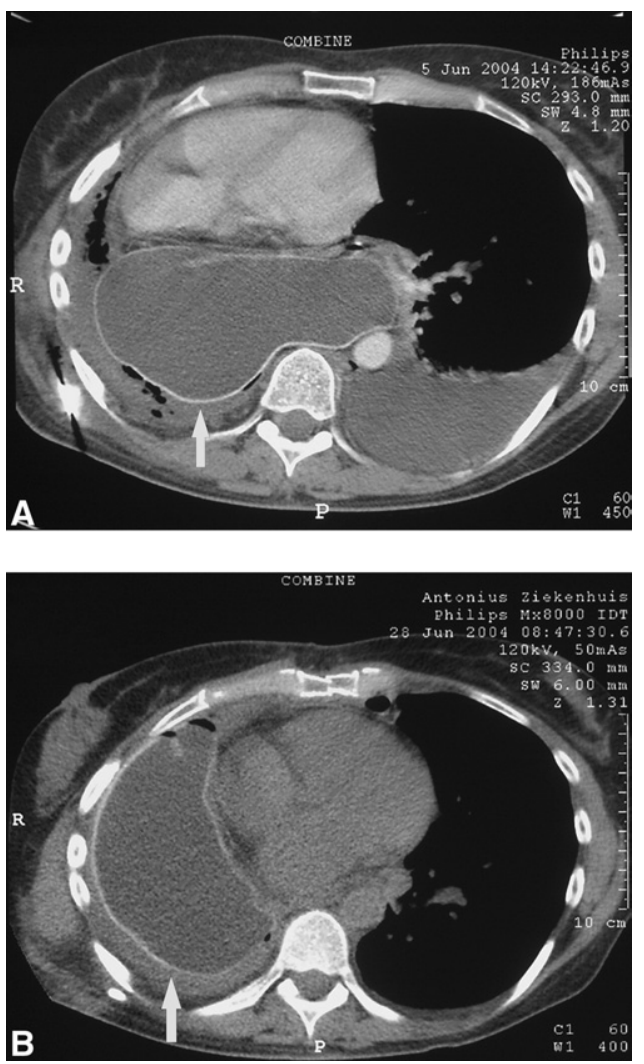

Figure 4. A, Postoperative computed tomographic scan demonstrates luxation of the tissue expander (arrow) behind the heart. B, Postoperative computed tomographic scan after replacement of the tissue expander (arrow). 

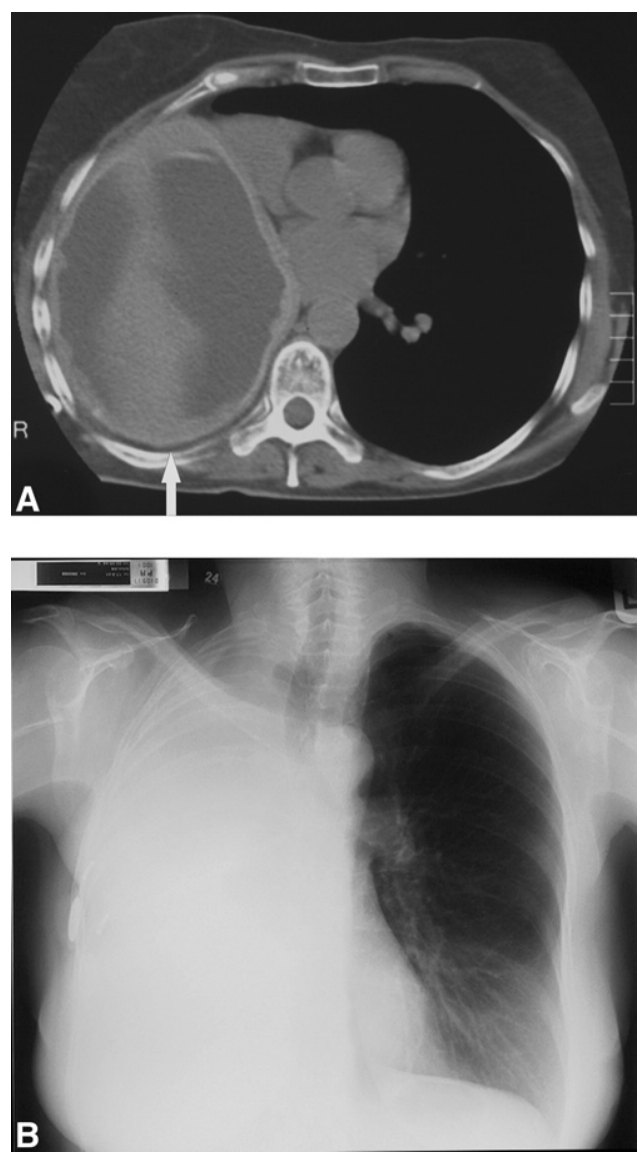

Figure 5. Computed tomographic scan (A) and chest X-ray film (B) 12 years after placement of the expandable prostheses (arrow).

such as inadequate position, herniation, and luxation, did not occur.

The patients did not have significant pain resulting from stretching of the pleura. Four patients visited the outpatient clinic with complaints of respiratory distress. Additional filling of the prostheses without surgical intervention improved lung function immediately.

Currently, the 12 surviving patients of the 19 treated are in good condition. Eleven of the survivors indicated by questionnaire not to have any limitation caused by respiratory distress, stridor, or complaints related to the treatment with TEs. They are now between 1 and 15 years postoperatively (Figure 5). The 7 other patients died of their initial disease.

\section{Discussion}

Although rare, PPS should be included in the differential diagnosis of progressive shortness of breath and recurrent infection after a pneumonectomy. The precise pathophysiology is not well elucidated, but because PPS is mostly seen in children, it is thought that elasticity and compliance of the remaining lung and mediastinum leads to shift and marked hyperinflation. ${ }^{1}$ This results in a twisting and narrowing of the left main bronchus, which adheres to the aortic arch. Recognition of PPS is critical in preventing malacic changes of the airway caused by prolonged compression.

Without treatment, PPS can be life-threatening. Management options for this syndrome are limited, and a review of the literature only reveals a few case reports and small series up to 11 patients. Various treatment strategies, such as aortic arch division with Dacron interposition, ${ }^{3}$ right phrenectomy to reduce thoracic volume, and filling of the empty hemithorax with saline and albumin, have been postulated. ${ }^{4}$ Endoscopic bronchial stent insertion has been reported to lead to correction of stridor. It is, however, not always possible to place a stent appropriately, particularly in the presence of gross distortion. Moreover, with this treatment, the cause of compression of the main bronchus, the displacement of the mediastinum, is not corrected. Stents should therefore only be considered in the management of patients with PPS who are not candidates for surgical intervention. ${ }^{5}$

The preferred treatment to date is the placement of TEs within the empty hemithorax to reposition the mediastinum to the midline and alleviate the bronchial obstruction. ${ }^{6-8}$

The advantages of using inflatable TEs include the uncomplicated procedure, involving minimally invasive surgical intervention. Progressive recentering of the mediastinum is possible, and decompression of the main bronchus can be observed immediately with bronchoscopy. Finally, the volume of the TEs can be increased postoperatively in an outpatient clinic setting, which means that the situation can be optimized in case of inadequate decompression or relapse of complaints. A new operation can be prevented in this way.

From 1990 onward, our center has used TEs as plombage in adult patients with PPS. ${ }^{9}$ Because we are a tertiary referral center, we have been able to compile the largest reported series of use of TEs to treat PPS. Our retrospective shows that TEs achieve lasting improvement of clinical symptoms (90\% of treated patients).

In patients with recurrent symptoms, the onset of a complication of implantation (ie, inadequate filling of or leaking from the TE, herniation, malposition, or luxation, all of which are readily correctable) must be excluded. In our opinion the position and status of the TE is best visualized with CT.

Because sternotomy for surgical release of the mediastinum will aggravate the procedure, this should only be used if centralization is not achieved by inflating the TEs after introduction through a thoracotomy or in complications caused by adhesions of the mediastinum. 
At present, the complication rate has been significantly reduced, partially because of improved quality of the TEs, leading to less leakage and shifting. An application to the TE to fix its position can be developed to prevent shifting of the TE.

In summary, pneumonectomy can, in rare cases, lead to a severe and life-threatening condition (PPS) caused by an obstruction of the left main bronchus. Insertion of TEs can enhance the clinical symptoms and reduce the functional obstruction. In our group the insertion of expandable prostheses dramatically reduced the functional obstructive syndrome and improved the clinical symptoms. In our opinion plombage with TEs is the most effective and easily performed means of solving this rare problem.

\section{References}

1. Rash DK, Grover FL, Schnapf BM, Clarke E, Pollard TG. Right pneumonectomy syndrome in infancy treated with an expandable prosthesis. Ann Thorac Surg. 1990;50:127-9.
2. Johnson J, Krirby C, Lazatin C, Cocke J. The clinical use of a prosthesis to prevent overdistention of the remaining lung following pneumonectomy. J Thorac Surg. 1949;18:164-72.

3. Szarnicki R, Maurseth K, de Leval M, Stark J. Tracheal compression by the aortic arch following right pneumonectomy in infancy. Ann Thorac Surg. 1978;25:231-5.

4. Wasserman K, Jamplis RW, Lash H, Brown HV, Cleary MB, Lafair J. Post-pneumonectomy syndrome: surgical correction using Silastic implants. Chest. 1979;75:78-81.

5. Cordova FC, Travaline JM, O'Brien GM, Ball DS, Lippmann M. Treatment of left pneumonectomy syndrome with an expandable endobronchial prosthesis. Chest. 1996;109:567-70.

6. Downey R, Trastek V, Clay R. Right pneumonectomy syndrome: Surgical correction with expandable implants. J Thorac Cardiovasc Surg. 1994;107:953-5.

7. Grillo H, Shepard J, Mathisen D, Kanarek D. Post-pneumonectomy syndrome. Diagnosis, management and results. Ann Thorac Surg. 1992;54:638-51.

8. Audry G, Balquet P, Vazquez MP, Sorrel Dejerine E, Baculard A, Boulé $\mathrm{M}$, et al. Expandable prosthesis in right postpneumonectomy syndrome in childhood and adolescence. Ann Thorac Surg. 1993;56:323-7.

9. Jansen JP, Brutel de la Rivière A, Carpentier Alting MP, Westermann CJJ, Bergstein PGM, Duurkens VAM. Postpneumonectomy syndrome in adulthood. Surgical correction using expandable prosthesis. Chest. 1992;101:1167-70. 